\title{
Surgery for Gastrointestinal Stromal Tumors: State of the Art of Laparoscopic Resection and Surgery for M1 Tumors
}

\author{
Ulrich Ronellenfitsch $^{a}$ Peter Hohenberger ${ }^{b}$

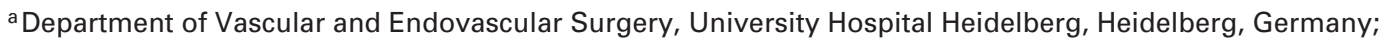 \\ ${ }^{b}$ Division of Surgical Oncology and Thoracic Surgery, Department of Surgery, Medical Faculty Mannheim of the University of Heidelberg, \\ Mannheim, Germany
}

\section{Keywords}

Gastrointestinal stromal tumor, GIST · Surgery · Laparoscopy · Metastatic stages

\section{Summary}

Background: The principles of surgery for primary gastrointestinal stromal tumor (GIST) are resection with clear margins without lymphadenectomy, thus enabling organ-preserving resection in many cases. Tumor rupture needs to be avoided, because it entails peritoneal sarcomatosis. Given these preconditions, there is a rationale for laparoscopic resection of primary GIST. In metastatic GIST, surgery cannot achieve cure, but constitutes an important cornerstone of treatment. Resection can be performed either for residual lesions in responding patients, for focally progressive lesions, or as palliative measure in generalized progression. Methods: Selective literature review. Results: There is ample evidence on laparoscopic resection of gastric GIST, but none from randomized trials. The studies show favorable perioperative outcomes and suggest adequate oncological results; however, a direct comparison to open resection is difficult. For surgery in M1 stages, survival outcomes are better for resection in generalized response than in focal progression. Perioperative morbidity is acceptable. Surgery in generalized progression does not prolong survival and bears relevant morbidity risks. Conclusion: Laparoscopy seems an adequate surgical approach for primary gastric GIST, as long as the surgical principles relevant for this entity are respected.
For other tumor sites, there is no sufficient evidence available. In M1 stages, selected patients may benefit from surgery. Survival differences between resection at different stages probably mirror the biology of the disease rather than the effect of the timing of the operation.

(c) 2018 S. Karger GmbH, Freiburg

\section{Introduction}

Surgery is an essential element in the treatment of gastrointestinal stromal tumor (GIST). For primary, non-metastatic GIST it constitutes the only potentially curative approach. With the advent of minimally invasive surgery for the resection of gastrointestinal malignancies, surgeons are faced with the question if laparoscopic resection is appropriate also for primary GIST. In metastatic (M1) stages of GIST, it complements systemic therapy in selected scenarios. This article reviews the underlying rationale and the available evidence for both laparoscopic resection of primary GIST and surgery for M1 GIST. To this end, a selective literature review was carried out.

\section{Principles of Surgery for Primary GIST}

Although GIST is a paradigmatic example for the efficacy of targeted drug therapy, the primary treatment and only option for cure in non-metastatic GIST remains complete surgical removal. Resection of GIST should follow the principles of oncological surgery, but certain biological properties of GIST alter some features of the

\section{KARGER}

() 2018 S. Karger GmbH, Freiburg 

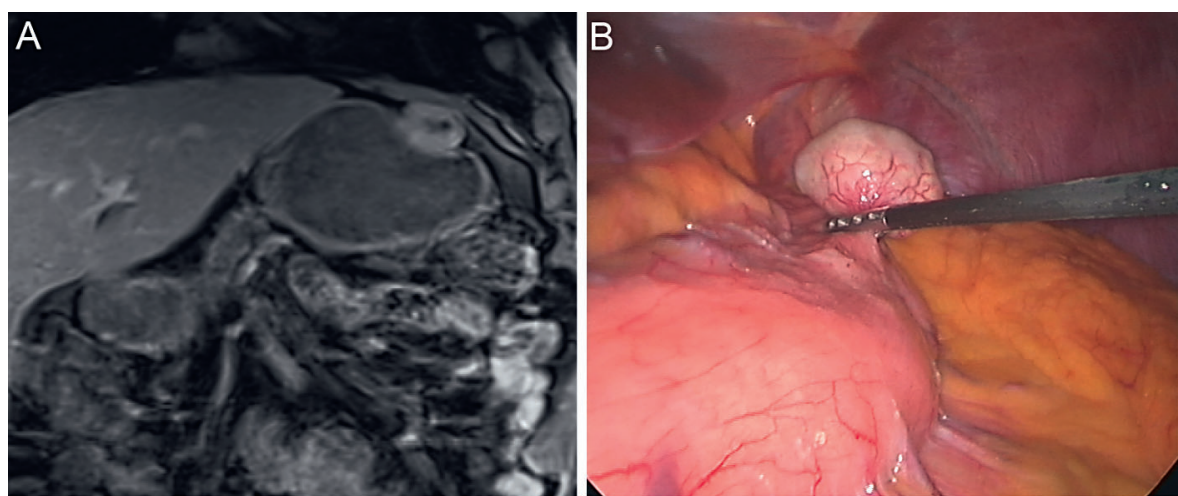

Fig. 1. Wedge resection of a lesser gastric curvature GIST (A). Organ-sparing resection is possible and no lymphadenectomy is warranted. The tumor is removed in sano using a linear stapling device (B, C). To avoid intra-abdominal tumor rupture or spillage of cells, use of a retrieval bag is mandatory (D) (from [27]; with kind permission from Georg Thieme Verlag KG)
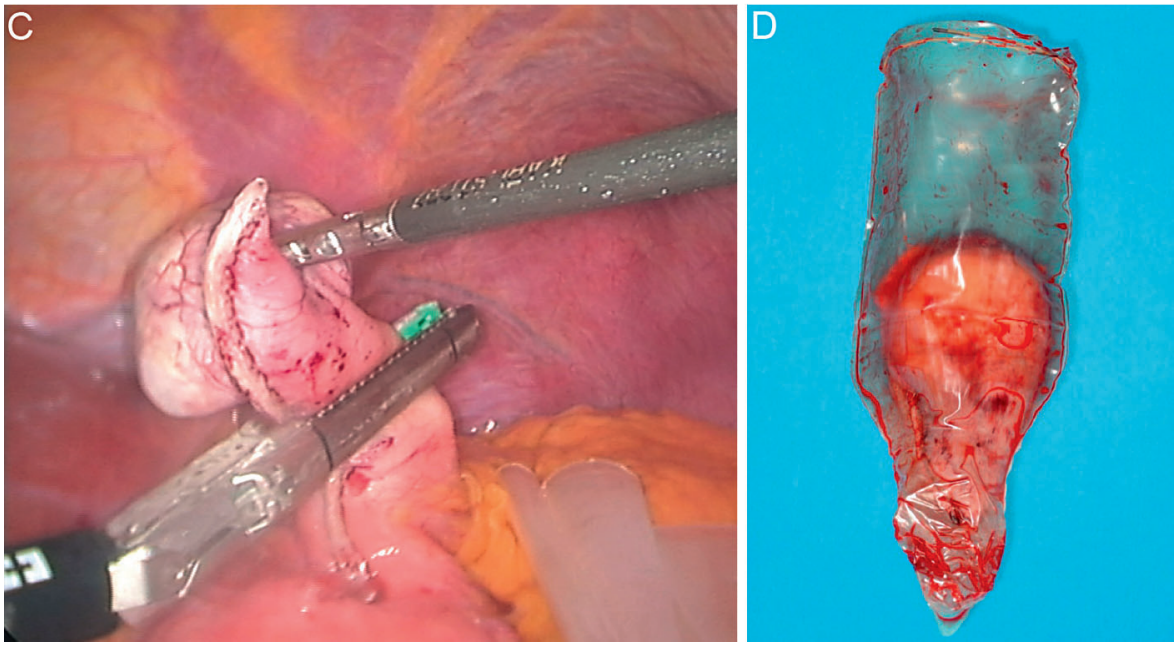

surgical approach compared to epithelial tumors, namely carcinomas, of the gastrointestinal tract. Like any malignant tumor, GIST should be resected with tumor-free margins. There is no uniformly accepted recommendation for the width of the resection margin one should aim at. Recent data suggest that a margin of $2 \mathrm{~cm}$ of tumor-free tissue towards the organ of origin, and intact adventitia, serosa, or peritoneum covering the tumor in other directions, are associated with longer survival compared to smaller margins [1]. In contrast to the surgical treatment of most carcinomas, whole organ removal (e.g. total gastrectomy, deep anterior rectal resection, hemicolectomy) is usually not required as long as a sufficient resection margin is guaranteed. This principle is based on the fact that lymphatic metastasis is an extremely rare event in GIST [2]. Therefore, systemic lymphadenectomy is not indicated and resection of the tumor-bearing part of the respective organ is sufficient. The only exception is constituted by the rare succinate dehydrogenase-deficient GIST, which are present in Carney-Stratakis syndrome [3]. In such cases, enlarged lymph nodes should be removed.

During resection of GIST, tumor rupture must be avoided at all cost. This is particularly important because GISTs are extensively neovascularized and are usually very fragile, which leads to a substantial rupture risk, which is reported to be up to $10 \%$. Rupture into the abdominal cavity has catastrophic sequelae as it almost inevitably leads to peritoneal sarcomatosis. Therefore, utmost caution must be exerted when handling the tumor intraoperatively [4].
If tumor rupture should still occur, or if a GIST has already ruptured spontaneously before surgery, the risk of recurrence might be lowered by extensive lavage of the abdominal cavity. Yet, there is no formal evidence supporting this approach. Moreover, preemptive imatinib therapy is strongly recommended in case of tumor rupture.

\section{Laparoscopy for Primary GIST}

\section{Principles}

Having the described surgical principles for resection of primary GIST in mind, surgeons are inevitably faced with the question if laparoscopic resection is an appropriate approach for GIST. Laparoscopy is now well established for the resection of many gastrointestinal carcinomas [5]. For certain stages of colorectal cancer, laparoscopic resection has replaced open surgery as the recommended approach. The pertaining evidence shows that it has undisputable benefits in terms of enhanced recovery, lower pain levels, and shorter hospital stay, while perioperative morbidity and mortality are at least equivalent to open resections. For colorectal cancer, there is higher-level evidence that the oncological outcome of laparoscopic resection is at least equivalent to that of open resection [6]. Therefore, a rationale for resecting GIST laparoscopically seems well justified. Tumor-free resection margins should be equally achievable by laparoscopy. Likewise, safe tumor handling 


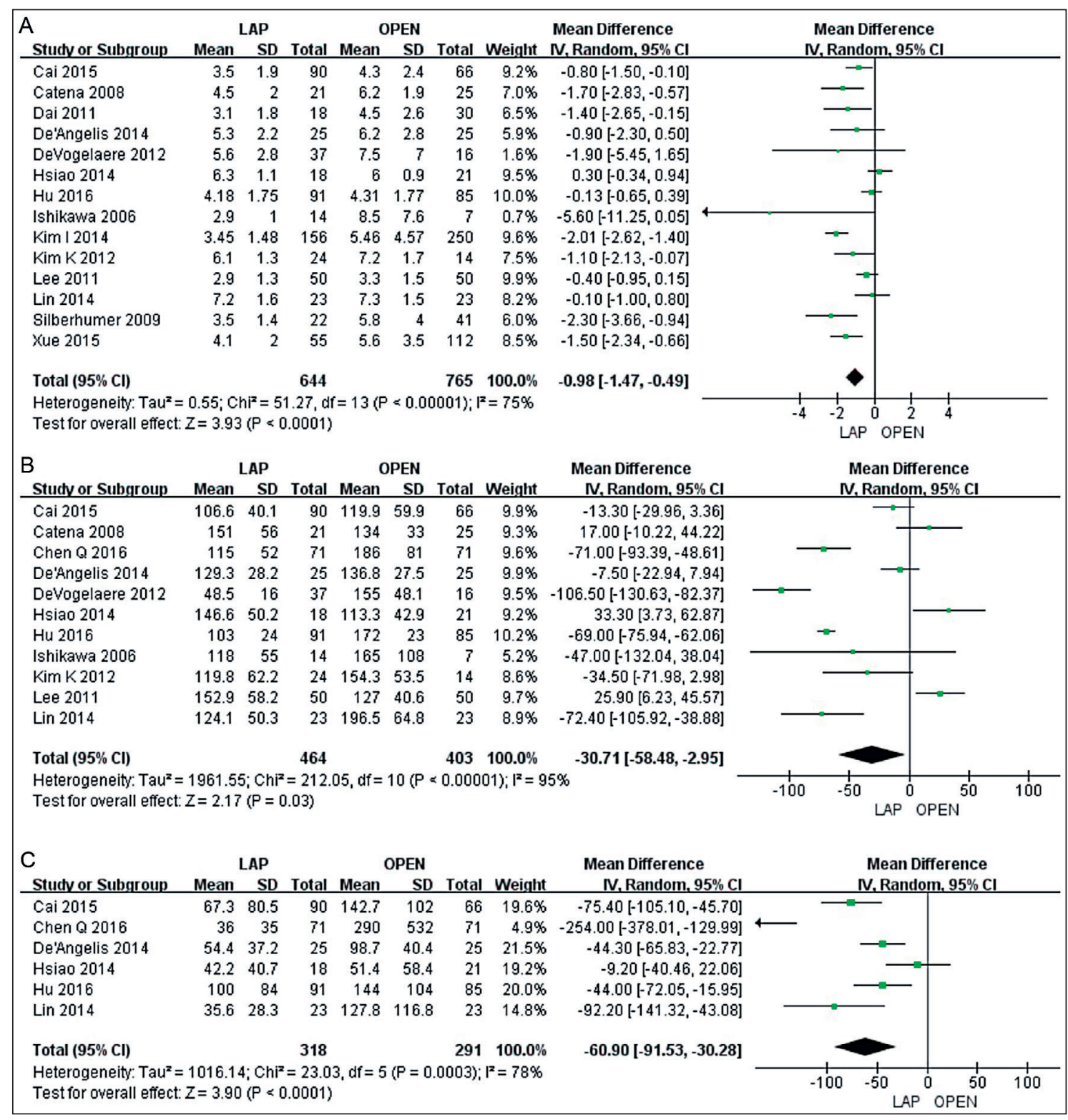

Fig. 2. Forest plots of the most recent meta-analysis comparing laparoscopic and open resection of GIST. A Tumor size, B operative time, $\mathbf{C}$ intraoperative blood loss (from [14]).

with the aim of avoiding tumor rupture can be expected if the surgeon has sufficient overall experience in laparoscopic surgery. As in open surgery, the tumor itself should not be touched during resection. For removal of the resection specimen, a retrieval bag is mandatory to avoid damaging the tumor surface when removing the specimen through port-site incisions (fig. 1). For the removal of large GIST, incisions would need to be considerably extended to allow removal of the specimen without morcellating the tumor, which is for obvious reasons to be strictly avoided. In such cases, the advantages of laparoscopy, which are mediated through the absence of incisions, would be offset. Consequently, in large tumors, even if laparoscopic resection seems technically possible, upfront open surgery should be preferred.

Given the anatomical peculiarities, most laparoscopic resections of GIST are carried out for gastric tumors. Gastric GIST can usually be resected without the need of creating an anastomosis by performing segmental or wedge resection with a linear stapling device. For small gastric GIST and gastric GIST in challenging locations such as the cardia or lesser curvature, hybrid endoscopic-laparoscopic procedures can be considered [7]. Such tumors are often hardly visible from outside the lumen. In such cases, the tumor can be exactly localized endoscopically, thus defining resection lines for the laparoscopic part of the operation. Moreover, particularly in cardial and subcardial locations, the endoscope or an endoscopically inserted thick gastric tube passing can help avoiding relevant narrowing of the gastric lumen by the resection.

For GIST of other locations, laparoscopic resection is carried out less often, due to both the relatively lower incidence and a higher 


\begin{tabular}{|c|c|c|c|c|c|c|c|c|c|c|c|c|}
\hline $\begin{array}{l}\text { A } \\
\text { Study or Subgroup }\end{array}$ & \multicolumn{2}{|c|}{$\begin{array}{l}\text { LAP } \\
\text { Events Total }\end{array}$} & \multicolumn{2}{|c|}{$\begin{array}{l}\text { OPEN } \\
\text { vents Total }\end{array}$} & \multicolumn{2}{|c|}{$\begin{array}{c}\text { Odds Ratio } \\
\text { Weight } \\
\text { M-H, Fixed, } 95 \% \mathrm{Cl}\end{array}$} & & \multirow{2}{*}{\multicolumn{4}{|c|}{$\begin{array}{c}\text { Odds Ratio } \\
\text { M-H, Fixed, } 95 \% \mathrm{Cl}\end{array}$}} & \\
\hline Cai 2015 & 4 & 90 & 8 & 66 & $8.2 \%$ & $0.34[0.10,1.17]$ & & & & & & \\
\hline Catena 2008 & 0 & 21 & 1 & 25 & $1.2 \%$ & $0.38[0.01,9.82]$ & & & & & & \\
\hline Chen Q 2016 & 4 & 71 & 16 & 71 & $14.0 \%$ & $0.21[0.06,0.65]$ & & & & & & \\
\hline Chen Y 2012 & 1 & 16 & 8 & 42 & $3.8 \%$ & $0.28[0.03,2.47]$ & & & & & & \\
\hline Dai 2011 & 1 & 18 & 10 & 30 & $6.6 \%$ & $0.12[0.01,1.01]$ & & & & & & \\
\hline De'Angelis 2014 & 1 & 25 & 5 & 25 & $4.5 \%$ & $0.17[0.02,1.55]$ & & & & & & \\
\hline Devogelaere 2012 & 1 & 37 & 3 & 16 & $3.8 \%$ & $0.12[0.01,1.26]$ & & & & & & \\
\hline Goh 2015 & 3 & 50 & 5 & 50 & $4.4 \%$ & $0.57[0.13,2.55]$ & & & & & & \\
\hline Hu 2016 & 9 & 91 & 16 & 85 & $13.8 \%$ & $0.47[0.20,1.14]$ & & & & & & \\
\hline Karakousis 2011 & 6 & 40 & 10 & 40 & $7.9 \%$ & $0.53[0.17,1.63]$ & & & & - & & \\
\hline Kasetsermwiriya 2014 & 1 & 23 & 1 & 10 & $1.2 \%$ & $0.41[0.02,7.27]$ & & & & & & \\
\hline Kim K 2012 & 1 & 24 & 0 & 14 & $0.5 \%$ & $1.85[0.07,48.55]$ & & & & & & \\
\hline Lee 2011 & 2 & 50 & 1 & 50 & $0.9 \%$ & $2.04[0.18,23.27]$ & & & & & & \\
\hline $\operatorname{Lin} 2014$ & 2 & 23 & 3 & 23 & $2.5 \%$ & $0.63[0.10,4.21]$ & & & & & & \\
\hline Melstrom 2012 & 2 & 17 & 4 & 29 & $2.4 \%$ & $0.83[0.14,5.11]$ & & & & & & \\
\hline Mochizuki 2006 & 3 & 12 & 4 & 10 & $3.0 \%$ & $0.50[0.08,3.08]$ & & & & & & \\
\hline Silberhumer 2009 & 0 & 22 & 3 & 41 & $2.2 \%$ & $0.24[0.01,4.95]$ & & & & & & \\
\hline Takahashi 2014 & 1 & 12 & 1 & 15 & $0.8 \%$ & $1.27[0.07,22.72]$ & & & & & & \\
\hline Wan 2012 & 4 & 68 & 20 & 88 & $15.2 \%$ & $0.21[0.07,0.66]$ & & & & & & \\
\hline Xue 2015 & 2 & 55 & 5 & 112 & $2.9 \%$ & $0.81[0.15,4.30]$ & & & & & & \\
\hline Total $(95 \% \mathrm{Cl})$ & & 765 & & 842 & $100.0 \%$ & $0.38[0.27,0.54]$ & & & & & & \\
\hline Total events & 48 & & 124 & & & & & & & & & \\
\hline $\begin{array}{l}\text { Heterogeneity: } \mathrm{Chi}^{2}=11 \\
\text { Test for owerall effect: } Z\end{array}$ & $\begin{array}{l}05, d f=19 \\
5.36(P<C\end{array}$ & $\begin{array}{l}(P=0 \\
0.0000\end{array}$ & 92); $\left.\right|^{2}=$ & & & & 0.01 & 0.1 & LAP $^{1}$ & OPEN & 10 & 100 \\
\hline B & LAP & & OPEN & & & Odds Ratio & & & Odds & Ratio & & \\
\hline Studv or Subgroup & Events & Iotal & Events & Total & Weight & M-H, Fixed, $95 \% \mathrm{Cl}$ & & & M-H, Fixe & d. $95 \% \mathrm{Cl}$ & & \\
\hline Cai 2015 & 2 & 90 & 2 & 66 & $2.8 \%$ & $0.73[0.10,5.30]$ & & & & & & \\
\hline Catena 2008 & 0 & 21 & 1 & 25 & $1.7 \%$ & $0.38[0.01,9.82]$ & & & & & & \\
\hline Chen Q 2016 & 6 & 71 & 5 & 71 & $5.7 \%$ & $1.22[0.35,4.19]$ & & & & & & \\
\hline Chen Y 2012 & 1 & 16 & 2 & 42 & $1.3 \%$ & $1.33[0.11,15.81]$ & & & & & & \\
\hline Dai 2011 & 2 & 18 & 3 & 30 & $2.5 \%$ & $1.13[0.17,7.47]$ & & & & & & \\
\hline De'Angelis 2014 & 1 & 25 & 2 & 25 & $2.4 \%$ & $0.48[0.04,5.65]$ & & & & & & \\
\hline Devogelaere 2012 & 0 & 37 & 6 & 16 & $11.1 \%$ & $0.02[0.00,0.41]$ & $\longleftarrow$ & & - & & & \\
\hline Goh 2015 & 0 & 50 & 4 & 50 & $5.6 \%$ & $0.10[0.01,1.95]$ & $\leftarrow$ & & & - & & \\
\hline Hsiao 2014 & 1 & 18 & 0 & 21 & $0.5 \%$ & $3.69[0.14,96.22]$ & & & & & & \\
\hline Hu 2016 & 12 & 91 & 17 & 85 & $19.2 \%$ & $0.61[0.27,1.36]$ & & & $\longrightarrow$ & - & & \\
\hline Ishikawa 2006 & 2 & 14 & 1 & 7 & $1.4 \%$ & $1.00[0.07,13.37]$ & & & & & & \\
\hline Karakousis 2011 & 1 & 40 & 1 & 40 & $1.2 \%$ & $1.00[0.06,16.56]$ & & & & & & \\
\hline Kasetsermwiriya 2014 & 0 & 23 & 1 & 10 & $2.5 \%$ & $0.13[0.01,3.61]$ & $\hookleftarrow$ & & & & & \\
\hline Kim | 2014 & 0 & 156 & 11 & 250 & $11.1 \%$ & $0.07[0.00,1.14]$ & & & & & & \\
\hline Kim K 2012 & 1 & 24 & 3 & 14 & $4.6 \%$ & $0.16[0.01,1.71]$ & & & & - & & \\
\hline $\operatorname{Lin} 2014$ & 2 & 23 & 3 & 23 & $3.4 \%$ & $0.63[0.10,4.21]$ & & & & & & \\
\hline Melstrom 2012 & 0 & 17 & 4 & 29 & $4.1 \%$ & $0.16[0.01,3.20]$ & $\longmapsto$ & & & & & \\
\hline Nishimura 2007 & 1 & 39 & 4 & 28 & $5.7 \%$ & $0.16[0.02,1.50]$ & & & & - & & \\
\hline Silberhumer 2009 & 0 & 22 & 4 & 41 & $3.9 \%$ & $0.19[0.01,3.60]$ & $\longleftarrow$ & & & & & \\
\hline Takahashi 2014 & 1 & 12 & 2 & 15 & $2.0 \%$ & $0.59[0.05,7.43]$ & & & & & & \\
\hline Wan 2012 & 3 & 68 & 4 & 88 & $4.2 \%$ & $0.97[0.21,4.48]$ & & & & & & \\
\hline Xue 2015 & 0 & 55 & 3 & 112 & $2.9 \%$ & $0.28[0.01,5.55]$ & & & & & & \\
\hline Total $(95 \% \mathrm{Cl})$ & & 930 & & 1088 & $100.0 \%$ & $0.45[0.30,0.66]$ & & & & & & \\
\hline Total events & 36 & & 83 & & & & & & & & & \\
\hline $\begin{array}{l}\text { Heterogeneity: } \mathrm{Chi}^{2}=18 \\
\text { Test for owerall effect: } \mathrm{Z}\end{array}$ & $\begin{array}{l}08, d f=21 \\
4.01(P<C\end{array}$ & $\begin{array}{l}(P=0 . \\
0.0001)\end{array}$ & $64) ; 1^{2}=$ & & & & 0.01 & 0.1 & $\operatorname{LAP}^{1}$ & OPEN & 10 & 100 \\
\hline
\end{tabular}

Fig. 3. Forest plots of the most recent meta-analysis comparing laparoscopic and open resection of GIST. A Perioperative complications, B recurrence (from [14]).

degree of technical difficulty. Tumors located in the small or large bowel usually require anastomosing the bowel after resection of the tumor. This can be done either by intracorporal suturing or stapling, which is an advanced laparoscopic technique, or by extracorporal suturing using a utility incision of variable size. For rectal GIST, even though lymphadenectomy by complete mesorectal excision is not needed, transanal stapling or coloanal hand-suturing must be performed. Both the guidelines of the European Society for Medical Oncology (ESMO) [8] and the National Comprehensive Cancer Network (NCCN) [9] foresee laparoscopic resection of select GIST in their respective current version. The ESMO guidelines generally state that possible laparoscopic resection needs to follow the principles of oncological surgery. It discourages laparoscopic resection in patients with large GIST, without stating a clear size limit, 
because the risk of rupture is deemed disproportionately higher in such cases. The NCCN guidelines suggest to consider laparoscopic resection for GISTs in favorable locations such as the anterior wall of the stomach, jejunum, and ileum. Like in the ESMO guidelines, it is emphasized that the same surgical principles followed in open resection of GIST such as complete resection, preservation of the tumor pseudocapsule and strict avoidance of tumor rupture be observed, and that a retrieval bag be used to remove the specimen. Furthermore, the NCCN guidelines suggest that laparoscopic resection could be feasible in other anatomic sites such as the rectum.

\section{Evidence on Laparoscopy for Primary GIST}

The first laparoscopic resection of a GIST has been reported in 1992 [10]. However, the tumor entity as such had not even been defined at the time, and the resection was performed inadvertently during laparoscopic cholecystectomy. At the beginning of the 21st century, the first case series of laparoscopically resected GIST were published [11-13]. They were limited to gastric GIST and most of them had no comparator group of patients who underwent open resection. These initial series showed the general feasibility of the approach with favorable perioperative (good operation times, quick postoperative recovery) and short-term oncological outcomes (high rates of complete resection, low risk of tumor rupture). Later studies were partially prospective, and many of them had a control group of patients who underwent open resection. However, because patients were purposely selected into one of the operative approaches due to the lack of randomization, the comparability of the 2 groups, especially regarding risk features such as tumor size, mitotic count, and tumor location, is limited. Several meta-analyses have cumulated the available evidence. The most recently published one included all studies published after 1997 which compared laparoscopic with open resection of GIST of any location [14]. In total, 24 studies comprising 2,140 patients fulfilled the inclusion criteria. All of them were either retrospective casecontrol or prospective cohort studies without a randomized control group. Moreover, only one of the studies comprised also GIST of non-gastric locations, namely 18 patients with small bowel GIST [15]. The analysis showed that the mean tumor size of the laparoscopically operated patients was significantly smaller (fig. 2), which mirrors the selective use of laparoscopy. Regarding the perioperative outcomes operative time, intraoperative blood loss (fig. 2), time to flatus and oral intake, and hospital stay, a significant and clinically relevant difference in favor of laparoscopy was found. Specifically, mean length of hospital stay was 3.4 days shorter in the laparoscopy group. For all mentioned results, a substantial degree of heterogeneity was observed. The meta-analysis also assessed perioperative complications and long-term oncological outcomes, with no relevant heterogeneity among results (fig. 3 ). The incidence of perioperative complications was significantly lower in the laparoscopy group ( $6.3 \%$ vs. $14.7 \%$, odds ratio 0.38 ). The analysis of tumor recurrence showed a lower recurrence rate in the laparoscopy group (odds ratio 0.45 ), but, besides the difference in tumor size, median follow-up was also 9 months shorter in the laparoscopy group.

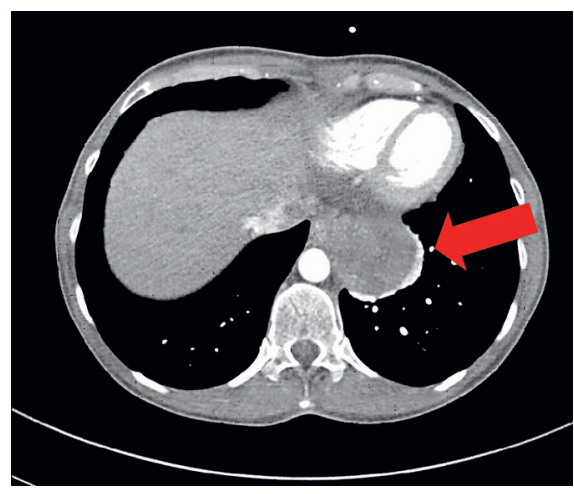

Fig. 4. Para-aortic locoregional recurrence of a GIST which was primarily located at the gastroesophageal junction. The recurrence was initially treated with imatinib for 6 months as mutational analysis confirmed an exon 11 mutation. During this time, no new tumor lesions appeared so that the patient underwent resection of the lesion (Ivor-Lewis esophagectomy with partial resection of the diaphragm and pulmonary wedge resection) and was rendered tumor-free. Postoperatively, imatinib treatment was continued.

Concerning laparoscopic resection of rectal GIST, the available evidence is very limited. The largest published series comprises 5 patients who underwent laparoscopic intersphincteric resection after neoadjuvant imatinib treatment [16]. All resections were complication-free and achieved tumor-free margins. None of the patients had recurred after a median follow-up of 36 months. An alternative minimally invasive approach for resection of rectal GIST is transanal endoscopic operation (TEO). This organ-preserving technique, in which transanal full thickness resection of the rectum is performed, can be used for circumscribed rectal GIST, usually after neoadjuvant imatinib treatment. Case series show promising oncological and functional results [17].

In summary, there is ample evidence demonstrating the feasibility and favorable short-term outcomes of laparoscopic resection of gastric GIST. Oncologic outcomes seem to be favorable, too. A direct comparison with open resection is, however, hampered by a complete lack of evidence from randomized trials and inherent differences between patients in the open and laparoscopic group in uncontrolled comparative studies. Regarding GIST of non-gastric location, there is hardly any evidence, so that valid conclusions cannot be drawn.

\section{Surgery for M1 GIST}

\section{Principles}

Once GIST has metastasized, either via a hematogenous route to the liver or through peritoneal spread to the abdominal cavity, neither surgery alone nor systemic therapy can achieve durable tumor control. However, a well-balanced combination of the 2 approaches can potentially create a stable disease situation and prolong overall survival to several years.

The large majority of patients shows a quick and sustained initial response to systemic therapy with tyrosine kinase inhibitors. How- 


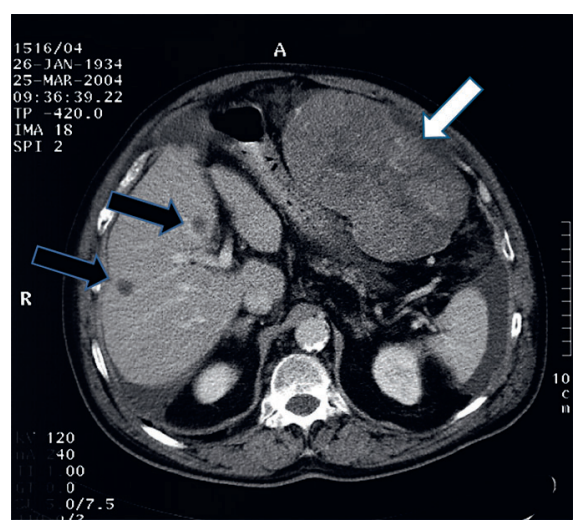

Fig. 5. Focal progression after 17 months of imatinib treatment. Large peritoneal lesion harboring a secondary mutation which confers resistance to imatinib (plain white arrow). The hepatic lesions (black arrows) still respond to imatinib.

ever, usually after 2-3 years of therapy, treatment resistance due to secondary mutations and subsequent tumor progression emerges. At present, the 3 kinase inhibitors imatinib, sunitinib, and regorafenib have a label for the treatment of metastatic GIST. Further drugs are being tested in clinical trials [18]. The common feature of all substances used for GIST treatment is that tumor progression occurs in one, few, or many locations after they have been administered for a certain amount of time. The typical pattern is that progression-free survival lasts long in early lines of treatment but is substantially shorter in later lines. When analyzing progressive lesions with molecular biology techniques, a secondary mutation conferring resistance to the specific drug can usually be demonstrated.

\section{Clinical Scenarios}

The described growth and response pattern of metastatic GIST entail indications for a surgical approach which differ from indications in gastrointestinal carcinomas. The possible scenarios are: resection of residual lesions in generalized response, selective resection of progressive lesions in focal progression, and resection/ debulking in generalized progression.

If a state of generalized response during systemic treatment is achieved, surgery has the potential to remove all macroscopic residual tumor manifestations (fig. 4). The underlying rationale is to decrease the risk of secondary mutations during continued systemic therapy by decreasing the number of tumor cells which are exposed to a stimulus to develop such mutations during treatment with kinase inhibitors. Even if all visible tumor manifestations can be resected, a discontinuation of systemic treatment must be strongly discouraged from because of the assumed presence of dormant micrometastases. Not surprisingly, most patients develop an early recurrence if therapy is stopped after complete resection of all tumor manifestations in generalized response. Therefore, one of the goals of surgery in this scenario is not to free the patient from the requirement of drug therapy but to preserve efficacy of the currently given systemic treatment as long as possible without the requirement need to switch to further lines due to secondary resistance.
As an alternative therapeutic strategy in metastatic GIST, focally progressive lesions can be selectively resected (fig. 5). Such lesions usually contain secondary mutations which yield resistance to the current systemic treatment [19]. By removing these lesions, the patient is given the possibility of staying on the current treatment and not having to escalate treatment to a more toxic second- or thirdline drug. Obviously, in analogy to generalized response, complete treatment discontinuation is not possible due to tumor lesions remaining in situ.

Patients with generalized tumor progression after multiple lines of systemic therapy often have a poor performance status and very limited life expectancy. At the same time, the tumor manifestations cause a considerable burden of symptoms. In these patients, surgery needs to be strictly limited to situations in which palliation seems achievable with an operation. Such scenarios include ileus, gastrointestinal or intra-abdominal hemorrhage, or metastasis-induced pain. Achieving a response to systemic therapy by means of decreasing the tumor burden is not a treatment goal in these patients. The expected benefit of an operation must be critically weighed against possible perioperative morbidity and mortality in this high-risk population.

\section{Perioperative Morbidity}

For patients with M1 GIST who undergo surgery, perioperative morbidity and mortality is of special concern. The respective patients often have a rather poor performance status and have received several lines of systemic therapy, which might both predispose to perioperative complications. However, in several studies both operations in patients with generalized response and in patients with focal progression were not associated with unusually high perioperative morbidity [20-22]. For debulking surgery in cases of generalized progression, however, the morbidity risk is higher, and the indication for surgery needs to be critically reflected [22].

Drugs used in the systemic treatment of GIST can affect tissue healing. Regarding imatinib, there is no evidence of impeded healing, and thus treatment with the drug does not need to be interrupted prior to surgery. In contrast, sunitinib and regorafenib are multikinase inhibitors and have other targets, amongst which is the vascular endothelial growth factor (VEGF). Its inhibition can severely disturb the healing of anastomoses and wounds. Therefore, treatment with sunitinib and regorafenib requires interruption early enough before the operation to ensure that the inhibition takes no longer place. Usually, a 1-week or longer drug-free interval is recommended prior to surgery. Postoperative therapy should only be reinitiated after all anastomoses and wounds have entirely healed, which is usually the case $2-3$ weeks after surgery [23].

\section{Survival}

When discussing surgery in M1 GIST, important outcomes are not only quality of life and a delay in escalation of systemic therapy but also possible effects on progression-free and overall survival. To date, no randomized controlled trials comparing surgery in the different described scenarios (generalized response vs. focal pro- 
Fig. 6. Overall survival, measured from the time of operation, after resection of metastatic GIST $\mathbf{A}$ in generalized response and $\mathbf{B}$ in focal progression, stratified by R0/1 and R2 resection (from [20]).

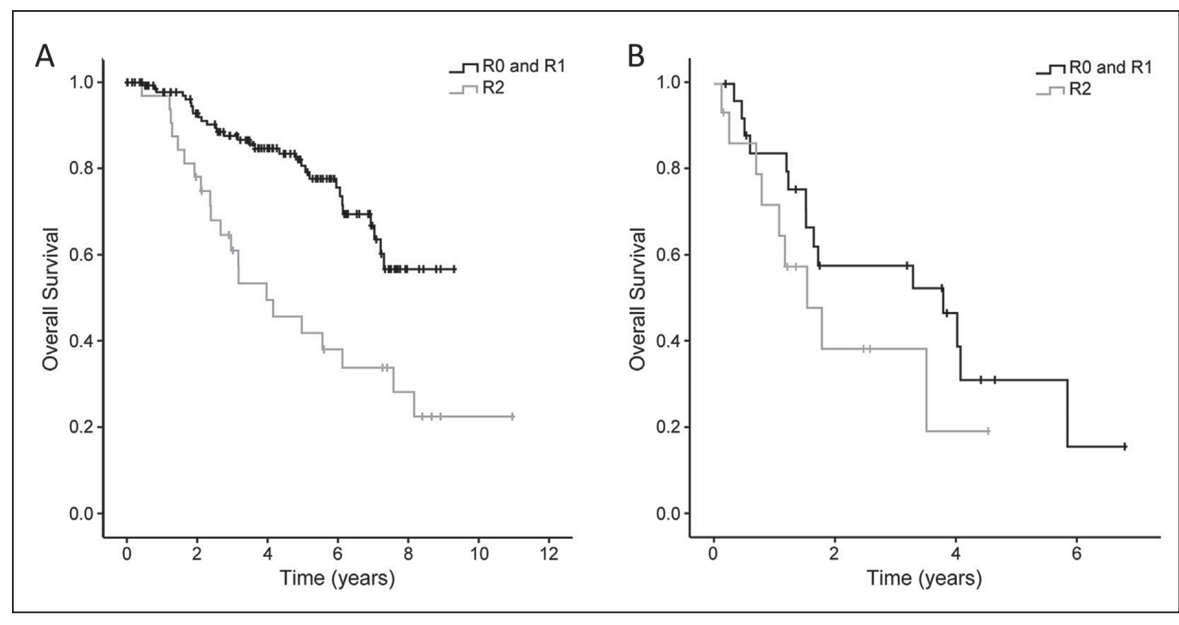

gression) or comparing surgery with medical therapy alone, have been conducted. Consequently, all available evidence stems from retrospective series with their inherent methodological limitations [20-22, 24-26]. All series come to relatively similar results. They are unable to demonstrate that surgery in either scenario prolongs survival compared to medical therapy alone. When comparing different clinical scenarios, however, survival after surgery in generalized response is significantly longer than after surgery in focal progression (fig. 6). Only some series have evaluated debulking surgery in generalized progression. Postoperative survival in the respective cohorts was very short. All survival results most likely mirror the overall dynamics of the underlying disease rather than the specific effect of surgery. This is particularly likely because none of the series provides a valid control group of similar patients whose only different feature is that they do not receive surgery in addition to their systemic treatment. These results show that the critical selection of M1 GIST patients for surgery is of utmost importance.

\section{Conclusion}

For primary GIST not exceeding a certain size, laparoscopy seems to be an adequate surgical approach, as long as the surgical principles relevant for this entity are respected. The available evidence is almost exclusively limited to gastric GIST. A direct comparison to open resection is difficult because of the non-randomized methodology of all available studies. In M1 stages, selected patients may benefit from surgery by achieving complete or partial tumor regression and avoiding escalation of systemic therapy. Differences in observed survival after resection at different metastatic stages probably mirror the biology of the disease rather than the effect of the timing of the operation. Surgery in a stage of generalized progression bears a relevant risk of morbidity, must be limited to symptom palliation, and requires a careful selection of patients.

\section{Disclosure Statement}

The authors declare that they have no conflict of interest.

\section{References}

1 Ahlen J, Karlsson F, Wejde J, Nilsson IL, Larsson C Bränström R: Wide surgical margin improves the outcome for patients with gastrointestinal stromal tumors (GISTs). World J Surg 2018;42:2512-2521.

2 DeMatteo RP, Lewis JJ, Leung D, Mudan SS, Woodruff JM, Brennan MF: Two hundred gastrointestinal stromal tumors: recurrence patterns and prognostic factors for survival. Ann Surg 2000;231:51-58.

3 Miettinen M, Lasota J: Succinate dehydrogenase deficient gastrointestinal stromal tumors (GISTs) - a review. Int J Biochem Cell Biol 2014;53:514-519.

4 Hohenberger P, Ronellenfitsch U, Oladeji O, Pink D, Strobel P, Wardelmann E, Reichardt P: Pattern of recurrence in patients with ruptured primary gastrointestinal stromal tumour. Br J Surg 2010;97:1854-1859.
Hamed OH, Gusani NJ, Kimchi ET, Kavic SM: Minimally invasive surgery in gastrointestinal cancer: benefits, challenges, and solutions for underutilization. JSLS 2014;18:e2014.00134.

6 Tanis PJ, Buskens CJ, Bemelman WA: Laparoscopy for colorectal cancer. Best Pract Res Clin Gastroenterol 2014;28:29-39.

7 Ntourakis D, Mavrogenis G: Cooperative laparoscopic endoscopic and hybrid laparoscopic surgery for upper gastrointestinal tumors: current status. World J Gastroenterol 2015;21:12482-12497.

8 Casali PG, Abecassis N, Bauer S, et al: Gastrointestinal stromal tumours: ESMO-EURACAN Clinical Practice Guidelines for diagnosis, treatment and follow-up. Ann Oncol 2018;DOI: 10.1093/annonc/mdy095.
9 National Comprehensive Cancer Network: Clinical Practice Guidelines in Oncology - Soft Tissue Sarcoma. Version 2.2018. www.nccn.org/professionals/ physician_gls/pdf/sarcoma.pdf.

10 Lukaszczyk JJ, Preletz RJ: Laparoscopic resection of benign stromal tumor of the stomach. J Laparoendosc Surg 1992;2:331-334.

11 Otani Y, Ohgami M, Igarashi N, Kimata M, Kubota T, Kumai K, Kitajima M, Mukai M: Laparoscopic wedge resection of gastric submucosal tumors. Surg Laparosc Endosc Percutan Tech 2000;10:19-23.

12 Hindmarsh A, Koo B, Lewis MP, Rhodes M: Laparoscopic resection of gastric gastrointestinal stromal tumors. Surg Endosc 2005;19:1109-1112. 
13 Mochizuki Y, Kodera Y, Fujiwara M, Ito S, Yamamura Y, Sawaki A, Yamao K, Kato T: Laparoscopic wedge resection for gastrointestinal stromal tumors of the stomach: initial experience. Surg Today 2006;36:341347.

14 Xiong H, Wang J, Jia Y, et al: Laparoscopic surgery versus open resection in patients with gastrointestinal stromal tumors: an updated systematic review and meta-analysis. Am J Surg 2017;214:538-546.

15 Chen YH, Liu KH, Yeh CN, Hsu JT, Liu YY, Tsai CY, Chiu CT, Jan YY, Yeh TS: Laparoscopic resection of gastrointestinal stromal tumors: safe, efficient, and comparable oncologic outcomes. Laparoendosc Adv Surg Tech A 2012;22:758-763.

16 Fujimoto Y, Akiyoshi T, Konishi T, Nagayama S, Fukunaga $Y$, Ueno M: Laparoscopic sphincter-preserving surgery (intersphincteric resection) after neoadjuvant imatinib treatment for gastrointestinal stromal tumor (GIST) of the rectum. Int J Colorectal Dis 2014; 29:111-116.

17 Han X, Xu J, Qiu H, Lin G: A novel curative treatment strategy for patients with lower grade rectal gastrointestinal stromal tumor: chemoreduction combined with transanal endoscopic microsurgery. J Laparoendosc Adv Surg Tech A 2017;27:579-585.
18 Wozniak A, Gebreyohannes YK, Debiec-Rychter M, Schöffski P: New targets and therapies for gastrointestinal stromal tumors. Expert Rev Anticancer Ther 2017;17:1117-1129.

19 Tamborini E: Mechanism of resistance in gastrointestinal stromal tumors; in Barrett JE (ed): Handbook of Experimental Pharmacologoy. Heidelberg, Springer, 2017.

20 Bauer S, Rutkowski P, Hohenberger P, et al: Long-term follow-up of patients with GIST undergoing metastasectomy in the era of imatinib - analysis of prognostic factors (EORTC-STBSG collaborative study). Eur J Surg Oncol 2014;40:412-419.

21 Mussi C, Ronellenfitsch U, Jakob J, Tamborini E, Reichardt P, Casali PG, Fiore M, Hohenberger P, Gronchi A: Post-imatinib surgery in advanced/metastatic GIST: is it worthwhile in all patients? Ann Oncol 2010; 21:403-408.

22 Raut CP, Posner M, Desai J, Morgan JA, George S, Zahrieh D, Fletcher CD, Demetri GD, Bertagnolli MM: Surgical management of advanced gastrointestinal stromal tumors after treatment with targeted systemic therapy using kinase inhibitors. J Clin Oncol 2006;24: 2325-2331.
23 Bose D, Meric-Bernstam F, Hofstetter W, Reardon DA, Flaherty KT, Ellis LM: Vascular endothelial growth factor targeted therapy in the perioperative setting: implications for patient care. Lancet Oncol 2010; 11:373-382.

24 DeMatteo RP, Maki RG, Singer S, Gonen M, Brennan MF, Antonescu CR: Results of tyrosine kinase inhibitor therapy followed by surgical resection for metastatic gastrointestinal stromal tumor. Ann Surg 2007; 245:347-352.

25 Rutkowski P, Nowecki Z, Nyckowski P, et al: Surgical treatment of patients with initially inoperable and/or metastatic gastrointestinal stromal tumors (GIST) during therapy with imatinib mesylate. J Surg Oncol 2006; 93:304-311.

26 Fairweather M, Balachandran VP, Li GZ, et al: Cytoreductive surgery for metastatic gastrointestinal stromal tumors treated with tyrosine kinase inhibitors: a 2-institutional analysis. Ann Surg 2018;268:296-302.

27 Hohenberger $\mathrm{P}$, Ronellenfitsch U: Chirurgische Therapie; in Messmann H, Tannapfel A, Werner J (eds): Gastrointestinale Onkologie. Stuttgart/New York, Thieme, 2018, pp 375-379. 